\title{
Faire face au racisme en France et au Brésil : de la condamnation morale à l'aide aux victimes
}

\section{Alexandra Poli}

\section{(2) OpenEdition}

\section{Journals}

Édition électronique

URL : http://journals.openedition.org/conflits/1875

DOI : $10.4000 /$ conflits. 1875

ISSN : $1777-5345$

Éditeur :

CCLS - Centre d'études sur les conflits lilberté et sécurité, L'Harmattan

Édition imprimée

Date de publication : 1 septembre 2005

Pagination : 11-45

ISBN : 2-7475-9476-9

ISSN : 1157-996X

Référence électronique

Alexandra Poli, «Faire face au racisme en France et au Brésil : de la condamnation morale à l'aide aux victimes », Cultures \& Conflits [En ligne], 59 | automne 2005, mis en ligne le 09 février 2006, consulté le 30 mars 2021. URL : http://journals.openedition.org/conflits/1875 ; DOI : https://doi.org/10.4000/ conflits. 1875

Ce document a été généré automatiquement le 30 mars 2021.

Creative Commons License 


\title{
Faire face au racisme en France et au Brésil : de la condamnation morale à l'aide aux victimes
}

\author{
Alexandra Poli
}

1 La lutte contre le racisme s'est considérablement développée et transformée ces dernières décennies. Influencée par l'essor de la mobilisation internationale autour de la notion de droits de l'Homme ${ }^{1}$, ses orientations et ses réalisations reposent avant tout sur la culture politique nationale dans laquelle elle s'inscrit. Les débats de la dernière conférence internationale contre le racisme qui s'est tenue en 2001 à Durban, témoignent d'ailleurs des difficultés à trouver un langage commun sur la question face à l'hétérogénéité des définitions suivant les pays, et les groupes d'individus concernés².

2 Prenons le cas du Brésil et de la France. Le mythe de la démocratie raciale au Brésil semble être à l'opposé du mythe français de la République. Le premier fait de l'harmonie des relations entre les races qui serait le fruit des multiples métissages ayant marqué l'histoire du Brésil un pilier de la démocratie. Ce récit, qui s'est construit progressivement depuis l'abolition de l'esclavage ne laisse guère de place à la reconnaissance du racisme dont l'existence même est sans cesse sujette à caution ${ }^{3}$. La part du racisme et la façon dont il se manifeste dans la société restent encore mal définies dans un pays dont près de $45 \%$ de la population est noire ${ }^{4}$.

3 Le mythe républicain français, imprégné de l'esprit des Lumières, prône quant à lui la mise à l'écart de tout particularisme culturel afin d'assurer le traitement égalitaire des citoyens. Les principes républicains garantissent une vigilance et une condamnation exigeantes du racisme tout en limitant le débat sur les catégories mêmes que le phénomène met en jeu. L'impossibilité de prendre en compte les différences fondées sur l'origine, l'ethnie, ou la religion dans les statistiques limite la production d'une vue d'ensemble et conditionne la reconnaissance des discriminations raciales.

4 Cette toile de fond, propre à chaque pays, nous donne une première idée des difficultés pour mesurer le racisme et le combattre. Elle permet entre autres, de mieux saisir la nature du décalage que l'on observe au Brésil comme en France entre le développement 
d'une législation, de diverses ressources judiciaires pour lutter contre le racisme et les discriminations et leur application. Ces instruments peuvent en effet rester relativement ignorés par les institutions comme par les individus supposés s'en saisir.

Des deux côtés de l'Atlantique, les modes de compréhension du racisme ont fait peser une sorte de déterminisme sur les victimes en les maintenant de diverses manières à distance du problème. L'expérience vécue des victimes est longtemps restée dans l'ombre des drames et des blessures du passé ou encore de la question sociale. L'aide directe aux victimes implique en effet la reconnaissance d'une actualité embarrassante des discriminations raciales qui remet en question le fonctionnement profond de la société, de ses institutions et risque de menacer les bases de sa tradition démocratique.

6 Au fil du temps, les grandes mutations qui se jouent au sein de ces deux sociétés ont façonné un nouveau regard sur le racisme et les discriminations raciales en poussant l'action publique à s'intéresser de plus en plus aux individus qui les subissent. En particulier, la vitalité des affirmations identitaires ethniques, culturelles et religieuses, observables au Brésil comme en France, donne une nouvelle épaisseur à la question du racisme.

7 De la dénonciation à la prise en charge de son expérience vécue, les grandes étapes de l'action antiraciste dans les deux pays révèlent les processus de construction et de déconstruction du tabou qui entourent la reconnaissance du racisme, largement influencés par leur culture politique nationale. Aujourd'hui, les anciens cadres normatifs ne suffisent plus à contenir la lutte contre le racisme, laquelle se déploie dans un espace traversé par de multiples lignes de tension s'échafaudant du global au personnel, et lancent un défi à l'action de l'Etat dans ce domaine.

Des pensées racialistes à la dénonciation du racisme

8 La mobilisation internationale qui naît au lendemain de la seconde Guerre Mondiale à travers la création des Nations Unies place au cœur de sa réflexion la lutte contre le racisme. Les auteurs de la charte des Nations Unies énoncent dans ce document historique que chacun, sans distinction de couleur, de sexe, de langue ou de religion, peut se prévaloir des droits de l'Homme et des libertés fondamentales. Cet engagement pris au niveau international participe d'une transition historique déterminante qui conduit les Etats à se saisir de la question de la race, des relations interraciales et du racisme, comme en témoignent les exemples français et brésilien. Le débat ne touche pas cependant de la même façon les deux pays, même si l'enjeu reste avant tout de détourner ou d'annuler les doctrines et les idéologies racistes qui doivent devenir taboues. Un premier chantier s'opère autour de la remise en question des conceptions scientifiques de la race dont le remodelage doit signer la rupture avec l'ancien système racial.

Le dédale de relations entre l'idée de race et de racisme

9 La découverte des horreurs nazies en Europe, puis les mouvements de décolonisation des années 1950 ébranlent les fondements du racisme scientifique et rendent indispensable leur démontage. De profondes mutations touchent alors l'utilisation du concept de «race » qui devient de plus en plus embarrassant. La déconstruction des postulats scientifiques qu'il a longtemps servis prend des voies distinctes selon les pays. Dans la sociologie américaine des années 1950, le travail de Park, inspiré de la tradition théorique de l'interactionnisme symbolique, marque un tournant décisif en élaborant la notion de " race " comme une catégorie sociale, symboliquement constituée, émergeant des relations entre groupes ${ }^{5}$. Cette perspective contraste en effet avec la 
théorie de l'assimilation des noirs qui prévaut jusqu'alors dans la sociologie de l'école de Chicago. La question raciale se rapporte désormais aux situations de conflit et de compétition entre groupes que les acteurs sociaux interprètent en termes de « race ". Cette nouvelle approche permet par ailleurs de se distancier des formes variées de réductionnisme psychologique ou biologique que porte le concept. «Les race relations [précise Park] ne sont pas tant les relations entre individus de races différentes qu'entre individus conscients de ces différences $»^{6}$.

11 L'enjeu est donc de jouer sur la signification et l'interprétation du terme plus que sur l'existence du phénomène. Cette analyse a exercé une influence fondamentale sur la conceptualisation de l'idée de « race » dans le monde anglo-américain.

Dans d'autres pays comme en France, la condamnation du racisme scientifique ne procède pas de cette dissociation. Le terme demeure en effet largement teinté des idéologies qu'il a inspirées, dans la mesure où il porte la reconnaissance de différences entre les individus, ce qui va à l'encontre de l'esprit de la République ${ }^{7}$. Cela conduit à le diluer dans l'idée que les races n'existent pas et à faire de son usage une affirmation raciste.

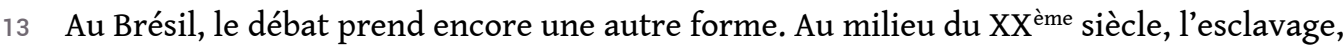
seulement aboli depuis 1888, imprègne encore largement les rapports sociaux. De plus, en pleine construction démocratique, l'Etat doit justifier des décennies de stratégies démographiques racialistes (idéologie du blanchiment) qui ont fait du métissage de la population sa spécificité. La politique volontaire de purification de la race, décidée par les élites politiques et intellectuelles tout au long du XIX ${ }^{\text {ème }}$ siècle est refondue dans une réflexion et une action d'ordre symbolique qui tend à magnifier le métissage ${ }^{8}$. Un peu dans le même sens qu'aux Etats-Unis et en Angleterre, on refond l'idée de race dans de nouvelles conceptions sans la rejeter totalement. A la nuance près qu'au Brésil, la différenciation entre les individus se fonde sur la couleur qui, tout en conservant une signification phénotypique, essentialiste, se rapportant à la peau, au type de cheveux, à la forme du nez et de la bouche, s'inscrit également dans leur statut social ${ }^{9}$. Ces réajustements traduisent en tout cas la volonté de canaliser, d'entourer la notion de race et de la dissocier du racisme.

L'enveloppe mythique des conceptions du racisme

14 L'idée de démocratie raciale au Brésil et le modèle républicain en France constituent la toile de fond des mouvements de composition et décomposition de la question du racisme dans les deux pays. Ces formules consacrées conditionnent d'une certaine façon l'existence du racisme et façonnent des impératifs bien distincts pour le combattre. Le cadre normatif dans lequel elles inscrivent l'harmonie entre les individus dans la société prend un caractère mythique en ce qu'il se fonde sur un récit idéalisé des relations sociales. Les expressions "pays des droits de l'Homme», et "terre du mélange des races " garantissent l'unité du peuple et excluent d'emblée l'existence du racisme. Cependant, d'un idéal à atteindre à ce qui passe pour acquis, du mythe à la réalité, les frontières se brouillent ou au contraire se durcissent et opèrent un jeu d'ombres et de lumières sur la question du racisme.

L'harmonie entre les races, pilier de la société brésilienne

Au Brésil, la conception mythique d'un pays sans discrimination raciale est enracinée dans une tradition de la pensée intellectuelle et politique sur le métissage. Déjà, dans les débats portés par les abolitionnistes, la stigmatisation liée à l'esclavage était bien plus comprise et critiquée en tant que symptôme de l'exploitation sociale 
qu'appréhendée comme destin racial ${ }^{10}$. Mais c'est surtout dans le milieu intellectuel des années 1930 que l'idée de l'harmonie entre les races va puiser sa force en servant la construction d'une identité nationale ${ }^{11}$. Les trois siècles de statut colonial, la persistance du code de l'esclavage et l'impact de l'idéologie du blanchiment, placent en effet la formation de la nation brésilienne et l'identité du peuple brésilien dans un questionnement permanent. Les idées portées par le courant littéraire moderniste du début $d u X X^{\text {ème }}$ siècle autour de ce thème reflètent l'effort pour trouver des réponses dans l'invention d'une nouvelle identité nationale, émancipée des modèles européens ${ }^{12}$. L'œuvre phare de Mario de Andrade, Macounaïma, ce " héros sans aucun caractère » à la recherche de son talisman perdu allégorise le Brésil en quête d'une identité. Au fil de continuels déplacements géographiques, langagiers, rituels, culturels, qui se croisent tout au long du récit, les aventures de ce personnage célèbrent le mélange des races et le syncrétisme de la culture brésilienne ${ }^{13}$. Le peuple brésilien est pluriel, composite et malgré tout uni pour constituer une seule et même nation. Ce mouvement de pensée trouve sa forme la plus aboutie dans les théories de l'anthropologue Gilberto Freyre à travers l'expression de « démocratie raciale $»^{14}$.

16 Comme le souligne Antonio Sergio Guimarães, Freyre parle d'abord de démocratie sociale et ethnique pour marquer le caractère positif du métissage de la population. Sa réflexion repose sur le fait que les colons portugais, en pratiquant des unions avec les populations indiennes, ont favorisé dès le début la production d'une société multiraciale. Plus encore, il définit les relations sociales comme méta raciales, c'est-àdire fonctionnant au-delà du concept de la race en raison d'une culture religieuse commune (la Chrétienté), et donc d'une fraternité donnant lieu à un syncrétisme avec les religions africaines centrées autour de la fête ${ }^{15}$. En faisant du mélange des races la particularité de la démocratie sociale dans le pays, Freyre tend à naturaliser le phénomène et à renverser les conséquences des logiques racialistes en principe social positif. Selon lui, l'intimité des relations entre le maître et l'esclave qui existe depuis des générations est censée annuler toute forme de racisme. De plus, le métissage de la population est érigé en indicateur de la tolérance entre les individus. En un sens, cette construction intellectuelle tend à effacer le caractère raciste de l'idéologie du blanchiment et empêche dans le présent et pour l'avenir un repérage potentiel du problème. Comme le soulignent Bastide et Fernandes, le mouvement noir des années 1930 ne s'éloigne guère de cette tendance en dénonçant l'exclusion sociale comme une conséquence du manque d'instruction et des coutumes archaïques de la population noire ${ }^{16}$. Il n'y a donc pas tant de victimes du racisme que du " préjugé de couleur » sur lequel pèse l'idée que les noirs sont surtout desservis par un faible niveau d'éducation.

Dans un premier temps, l'enjeu pour le Brésil est de se constituer en société démocratique moderne et de déployer une identité dans laquelle vient s'inscrire implicitement l'idée que le racisme ne peut exister. Même s'il s'agit là d'une construction intellectuelle, d'une mise en récit, cette vision se fond dans l'imaginaire national et dans les faits, la mobilisation contre le racisme reste faible. Contrastant considérablement avec le désastre de l'Europe, l'idée de démocratie raciale fascine d'autant plus et donne des raisons d'espérer une meilleure forme de civilisation ${ }^{17}$. Le mouvement noir fonde essentiellement ses revendications sur le registre de l'exploitation sociale et reste relativement éloigné de la question raciale. Le récit de la démocratie raciale s'enracine ainsi de plus en plus dans le réel, ménagé par un langage de précaution et de justification autour de la bonne entente naturelle entre les races qui maintient la catégorisation raciale dans une conception positive. Ces idées sont 
largement diffusées durant la période de l'Estado Novo (1937-1945), le régime autoritaire de Vargas. Dans les années 1940, Donald Pierson montre ainsi que les conflits à Bahia prennent surtout la forme d'antagonismes de classes et non d'antagonismes raciaux, et souligne qu'il existe une tendance à voir un conflit racial, dans des situations où c'est en réalité la compétition entre classes qui est en jeu ${ }^{18}$. Le problème n'est donc pas de comprendre les relations de pouvoir et d'inégalité en termes de différence raciale, mais plutôt de les considérer comme le produit des relations entre classes.

La catégorisation raciale, fondée sur la couleur, ne joue qu'après coup, dans la mesure où elle est déterminée par la situation sociale des individus. Le «blanc » et le « noir » correspondent à des positions socio-économiques, à tel point que la mobilité ascendante redéfinit la couleur de peau de l'individu. C'est pourquoi, comme le note Pierson, "branco » (blanc) renvoie à une personne blanche mais indépendamment de son origine raciale, incluant tous ceux qui, métis ou noirs, appartiennent aux classes supérieures. La désignation "preto " se réfère aussi bien à des traits physiques, qu'au fait d'avoir un bas statut social. Il n'empêche que la mobilité sociale est faible et que les noirs restent en majorité en bas de l'échelle sociale.

Au fil du temps, la lecture sociale des relations raciales se renforce pour des raisons à la fois d'ordre politique et idéologique. Dans la seconde moitié des années 1940, Gilberto Freyre consolide le mythe de la démocratie raciale en posant le métissage comme la garantie de l'unité du peuple brésilien. Dans un contexte politique international encore secoué par le fascisme, il saisit l'opportunité de façonner une expression démocratique inédite se démarquant des valeurs occidentales et s'opposant avec les conceptions défendues par le mouvement de la négritude. Parallèlement, les études en sciences sociales, largement influencées par le paradigme de la lutte des classes, insistent sur le caractère social des inégalités.

20 Le mythe de la démocratie raciale, tout en valorisant les différences qui ressortent du métissage s'inscrit dans une perspective universalisante. Il opère une dissociation entre une dimension raciale idéalisée et une dimension sociale perçue comme la cause du préjugé de couleur ressenti par la population noire. Les sciences sociales, alors imprégnées de la pensée marxiste, mais également soucieuses de se distancier des doctrines racialistes abondent dans ce sens. En 1957, Bastide insiste sur le fait qu'il n'existe pas au Brésil de conscience de race, ni de volonté des noirs de progresser collectivement ${ }^{19}$. Les conclusions de la vaste enquête parrainée par l'UNESCO au début des années 1950 sur la question raciale (à laquelle Bastide contribua d'ailleurs) soulignent la volonté de se débarrasser du tabou lié au problème racial, historiquement déterminé par la colonisation et l'esclavage. Les analystes mobilisés pour cette recherche apparaissent largement guidés par l'idéologie de la démocratie raciale. Sans annuler l'existence du préjugé racial, et plus précisément du préjugé de couleur, ils développent un nuancier assez large de constats qui révèle leurs précautions à parler de racisme. Dans les conclusions de l'enquête menée dans le Brésil rural et le Nordeste, Bastide affirme : «Le préjugé racial existe bien au Brésil (...) mais il se distingue de celui des Etats-Unis, parce qu'il ne discrimine pas les individus selon leurs origines ethniques, mais selon leur apparence physique - ce qui fait que la couleur peut être compensée par d'autres avantages, économiques par exemple. (...) Mais aujourd'hui, il n'existe pratiquement plus de préjugés ; si la couleur compte, certes, dans la classification des individus, une position sociale élevée est 
capable d'en diminuer les effets. La discrimination par contre, existe, mais elle est autant sociale et économique que raciale $»^{20}$.

21 La réflexion qui s'organise à l'époque autour de la question raciale affiche certes la volonté de faire émerger la problématique des discriminations raciales mais tout en la présentant d'emblée maîtrisée et contenue dans la pensée de la lutte des classes et de l'idéologie de la démocratie raciale. Cette perspective, on s'en doute, ne laisse guère d'espace aux revendications identitaires du mouvement noir. Le débat se polarise dans cette période sur l'existence ou non du " préjugé racial », autrement dit sur la frontière entre imaginaire et réel de la démocratie raciale. Jusqu'aux années 1960 au Brésil, l'enjeu n'est pas tant de remettre en question l'idée de démocratie raciale que de situer le racisme par rapport à celle-ci, ce qui donne d'office au phénomène une part relativement négligeable.

22 Cependant, les positions commencent à se durcir au fil du temps et diverses voix s'élèvent pour dénoncer la supercherie de ce que certains qualifient désormais de "mythe de la démocratie raciale», présenté comme un instrument au service de la suprématie blanche. Florestan Fernandes dénonce la manipulation de la démocratie raciale comme un lien dynamique des mécanismes de défense de la "race dominante " dissimulant des attitudes, des comportements et des idéaux "aristocratiques ${ }^{21}$. La démocratie raciale apparaît alors comme le berceau idéologique des discriminations que subissent les noirs et les métis, qui auraient pu l'exploiter à leurs propres fins. Cependant, cette critique du principe d'égalité entre les races est noyée par l'avènement de la dictature, qui décrète dans les actes institutionnels de 1964, "l'interdiction sociale de parler du racisme $»^{22}$.

Le modèle républicain d'intégration : du mythe garant de la lutte contre le racisme à l'injonction à l'intégration

23 En France, le mythe démocratique qui influence les conceptions du racisme paraît très éloigné du contexte brésilien. La période d'après-guerre plonge le pays dans une transition historique taraudée par le paradigme du génocide juif. Si au Brésil le débat se situe essentiellement au niveau intellectuel, il se développe plus en France sur le terrain politique et social. La lutte contre le racisme consiste surtout à inscrire dans les textes constitutionnels la notion de droits de l'Homme. Imprégnée de l'esprit républicain, la condamnation du racisme passe par une mise à l'écart des particularismes culturels, ethniques et religieux dans l'espace public. L'harmonie au sein de la société repose ainsi sur un principe d'égalité conditionné par l'idéal de l'effacement des différences. Dans la droite lignée de la déclaration des droits de l'Homme et du citoyen de 1789, la Constitution du 4 octobre 1958 stipule dans son article premier que «la France est une République indivisible, laïque, démocratique et sociale. Elle assure l'égalité devant la loi de tous les citoyens sans distinction d'origine, de race ou de religion. Elle respecte toutes les croyances ».

24 Sur le versant social, diverses associations, telles la Ligue Internationale contre le Racisme et l'Antisémitisme (LICRA), la Ligue des Droits de l'Homme et le Mouvement contre le Racisme et pour l'Amitié entre les Peuples (MRAP) se constituent ou poursuivent leur action dans le cadre de la résistance au nazisme. Dans la période d'après-guerre, ces associations se présentent comme autant de cellules de veille pour la sauvegarde des libertés individuelles et le respect de la dignité humaine. Dans le même temps, la décolonisation "dégéographise » en partie la question du racisme. La Ligue des Droits de l'Homme se consacre par exemple aux problèmes liés à la 
décolonisation et aux attitudes répressives et attentatoires aux droits de l'Homme des $\mathrm{IV}^{\text {ème }}$ et $\mathrm{V}^{\text {ème }}$ Républiques dans les colonies.

A la suite de cette période, marquée par le génocide de millions de juifs, les vagues d'immigration des années 1960 et 1970 ainsi que l'épisode de la guerre d'Algérie vont progressivement transformer l'espace du racisme. Au lendemain de la seconde Guerre Mondiale, le recours à l'immigration devient un objectif politique prioritaire pour les pouvoirs publics : il faut d'une part augmenter le volume de population active pour la reconstruction, et d'autre part, organiser une immigration de peuplement pour pallier le déficit démographique. La figure de l'immigré en tant que victime avérée ou potentielle du racisme apparaît uniquement dans l'espace public à travers des épisodes ponctuels et spectaculaires. Tel est notamment le cas de la flambée raciste de 1973 qu'Yvan Gastaut considère comme le phénomène où les Français furent confrontés pour la première fois depuis l'entre-deux-guerres à l'immigration comme un phénomène négatif. Outre les conséquences de la crise économique de 1972, cette flambée raciste serait «une forme de rancoeur liée à la guerre d'Algérie, apparue dans une partie de l'opinion $»^{23}$. A travers l'écho des médias et de la presse, le racisme dévoilé de certains Français devient un véritable problème de société. Bien qu'on ne puisse réduire la question du racisme aux conséquences de la guerre d'Algérie, les campagnes racistes repérables dès la fin des hostilités font du travailleur immigré algérien la principale cible du racisme. Les événements marquants qui s'échelonnent tout au long des années 1970 - on parle alors d'attentats racistes - sont rapportés aux séquelles du colonialisme, à une « violence néo-colonialiste contre les immigrés $»^{24}$.

Benjamin Stora considère ainsi que «les Maghrébins, en fait principalement les Algériens en France, peuvent d'autant plus être objets de répulsion qu'ils rappellent par leur présence la dernière guerre que la France a livrée (et perdue), cause d'une blessure nationale jamais refermée $»^{25}$.

Sur le plan des politiques publiques, l'immigration renvoie au besoin de main d'œuvre pour le pays ce qui la définit avant tout comme un enjeu socio-économique. Diverses institutions spécialisées sont créées pour venir en aide aux immigrants et améliorer leurs conditions de vie, telles le Fonds d'Action Sociale (FAS) en $1958^{26}$. En contraste avec la situation britannique, où l'enjeu de l'immigration renvoie à la question des différences raciales et religieuses des anciens colonisés, la France considère avant tout le phénomène en termes de main d'œuvre.

28 Au tournant du mois de juillet 1974, la suspension officielle de l'immigration en France met fin à la demande de main d'œuvre qui caractérisait et justifiait la venue et la présence de populations étrangères. La mutation qui s'opère dans le passage d'une immigration de main d'œuvre, perçue comme provisoire à une immigration définitive, familiale et de peuplement devient un enjeu majeur sur la scène politique et donne lieu aux premiers discours sur la "nécessaire intégration" des intéressés et de leurs descendants. En 1976, le gouvernement décide de reconnaître aux étrangers installés légalement en France le droit au regroupement familial.

29 Dans le même temps, apparaissent des manifestations ponctuelles et spectaculaires de racisme envers ces populations. Selon Gastaut, le parallèle est étroit entre la médiatisation de l'immigration et l'interrogation sur le racisme à l'œuvre dans la société française. La question « Sommes nous racistes? » est régulièrement soumise à l'opinion entre 1970 et $1983^{27}$. C'est notamment entre 1970 et 1973 que cette interrogation s'intensifie autour d'évènements dramatiques caractérisés par des faits 
divers et par une montée de violence contre les immigrés. Le lien avec la guerre d'Algérie ne fait nul doute. En 1971, après que le gouvernement algérien eut décidé de nationaliser ses compagnies pétrolières, une violente campagne anti-immigrés se développe. En 1973, une explosion, revendiquée par un mouvement d'extrême droite, composé d'anciens membres de l'OAS, dévaste le consulat d'Algérie à Marseille ${ }^{28}$. Les évènements qui s'échelonnent tout au long des années 1970 traduisent le passage d'un racisme colonial à un racisme anti-maghrébin qui, tout en s'inscrivant sur la toile de fond des conséquences de la crise économique, puise dans les rancœurs par rapport à la guerre d'Algérie ${ }^{29}$.

Au début des années 1980, la montée du chômage et les crises urbaines mettent en relief la précarité de la cohésion sociale et la marginalisation de la jeunesse issue de l'immigration. Des incidents fortement médiatisés dans les zones urbaines à problèmes où sont concentrées les populations immigrées ont conduit «la priorité de l'action publique à se focaliser sur ces lieux d'habitation $»^{30}$. Toujours est-il que la problématique de l'insécurité urbaine et de la délinquance croise largement le débat sur l'immigration regroupant dans une seule et même expression les immigrants et leur descendance que certains discours comme ceux du Front national rendent responsables de tous les maux de la société.

31 En 1983, un mouvement conduit par les jeunes de la deuxième génération s'attaque alors frontalement à ces confusions en articulant la dénonciation du racisme à l'hypocrisie du modèle d'intégration. En 1983, la «marche pour l'égalité et contre le racisme", (également appelée "marche des beurs») est l'occasion de montrer l'inconsistance des promesses de la République à l'égard des descendants d'immigrants maghrébins. Les parents sont présentés comme les victimes passives d'un racisme que les jeunes générations somment de regarder désormais en face tout en faisant valoir le droit à la différence.

Tout au long des années 1980, se développe dans l'espace public français le thème de la montée du racisme. En 1985, la création de l'association SOS racisme va entraîner une médiatisation croissante de la question. Cette nouvelle force antiraciste, à laquelle s'ajoutent Radio Beur, France Plus et bien d'autres, se démarque des canaux plus traditionnels de la lutte contre le racisme en affirmant l'implication de toute une génération qui s'organise dans un premier temps autour du débat sur le droit à la différence.

Cette tendance fait ressortir un mouvement de "victimisation positive » qui consiste à faire du racisme un enjeu de société tout en construisant une nouvelle image des jeunes de la seconde génération déterminés à prendre la parole, à se donner une visibilité dans l'espace public en mettant en avant le multiculturalisme et le métissage de la France. Plus encore, il s'agit de remettre en question les représentations concernant l'égalité des droits telles que portées par les principes républicains ${ }^{31}$.

Cependant, si la question du racisme est revisitée un temps par ces nouvelles revendications, le mouvement qui leur est associé s'effrite progressivement et le débat s'ankylose rapidement sur ce qui devient le problème de l'immigration. La lutte contre le racisme se poursuit certes sur le plan associatif, notamment par le biais de SOS racisme mais se range du côté du thème de l'intégration et se détache du volet identitaire. Tout au long des années 1980, les acteurs politiques et associatifs définissent les tensions racistes comme la manifestation des problèmes sociaux dans les quartiers d'exclusion. La question sociale est alors déterminante et noie les individus, 
victimes ou auteurs du racisme, dans la même équation des difficultés du vivre ensemble dans les banlieues.

Ainsi, il existe moins de victimes du racisme que de victimes des inégalités sociales, ce qui conduit en retour à envisager l'intégration socio-économique des individus comme la fin du racisme. Au fond, au-delà des raisons structurelles invoquées, comme le chômage, l'insalubrité de l'habitat, le principal coupable identifié sans ambages est le Front national dont les scores se font de plus en plus menaçants vers la fin des années 1980. Ces résultats inquiétants poussent la lutte contre le racisme dans les années 1990 à faire du parti son ennemi principal.

Sur le plan des recherches universitaires, la question du vécu du racisme ne ressort que par touches dans le cadre des travaux portant sur les formes d'exclusion ${ }^{32}$ et la vie dans les quartiers populaires, articulée à diverses problématiques telles que la violence, l'islam des jeunes, l'école etc. La question du racisme apparaît le plus souvent en filigrane des analyses sur l'univers de la banlieue dont les expressions se multiplient. Les "quartiers d'exil »"33 sont le cadre des manifestations du racisme, souvent décrit comme le dernier pilier auquel «les petits blancs» se raccrochent et sur lequel viennent se cristalliser des perceptions d'insécurité multiples et entremêlées, que l'on se place du côté des racistes ou de ceux qui en sont la cible.

La lutte contre le racisme dans la coulée des mythes : le racisme dans l'ombre de la question sociale

Malgré l'émergence de nouvelles revendications qui viennent dénoncer l'imposture ou l'hypocrisie des mythes, les politiques publiques française et brésilienne restent axées dans un premier temps sur ces cadres normatifs pour définir le combat contre le racisme. Au Brésil, la fin des années 1980 marque cependant un pas décisif pour la reconnaissance du racisme à travers sa criminalisation. En même temps, on insiste sur la force du récit de la démocratie raciale dans l'imaginaire collectif, ce qui traduit une tension entre le renforcement du dispositif légal et les logiques personnelles et sociales, ou encore entre le niveau politique et le niveau des pratiques.

Est-ce que le racisme existe si personne ne le reconnaît comme tel ?

38 Les mouvements politiques noirs brésiliens qui ressurgissent à la fin des années 1970 s'emparent du thème de l'imposture du mythe de la démocratie raciale, défendu par Florestan Fernandes dès le milieu des années 1960. De même, une nouvelle génération de chercheurs met l'accent sur la façon dont certaines formes de discriminations raciales structurent les inégalités ${ }^{34}$. Tout au long des années 1980, "le mythe de la démocratie raciale » est systématiquement dénoncé comme un dogme de la «suprématie blanche $»^{35}$. Fondé le 18 juin 1978 à São Paulo, le Mouvement Noir Unifié (MNU) dénonce à la fois la manipulation de la culture noire ainsi que les logiques étatiques qui font du noir un sous-citoyen. Ces deux revendications traduisent d'ailleurs les tendances actuelles qui caractérisent le mouvement noir, partagé de manière très classique entre une sensibilité égalitaire, porteuse de demandes de discrimination positive à l'égard de la population noire, et une sensibilité plus culturaliste ${ }^{36}$. Même si l'influence de ces mouvements a souvent été remise en question par le constat dominant du manque de protestation des Afro-brésiliens ${ }^{37}$, leurs demandes représentent un élément essentiel de la toile de fond sur laquelle se renouvelle la réflexion concernant les termes de l'appartenance nationale ${ }^{38}$.

39 Les années 1980 marquent un réel changement dans l'approche des questions ethniques et raciales par les autorités brésiliennes. A l'exception d'une loi de 1951, punissant la 
discrimination raciale, il n'existe pas jusqu'alors de véritable corpus législatif pour lutter contre le racisme. De plus, la mise en pratique des lois reste le plus souvent en suspens étant donné que la police reçoit très peu de plaintes et qu'il y a peu de procès pour des affaires de racisme ou de discrimination raciale. Selon le ministre de la Justice, le racisme se manifesterait le plus souvent par des attaques verbales ou des injures qui ne donneraient pas lieu à des plaintes. Eccles note d'ailleurs qu'entre 1951 et 1991, seuls trois cas ont été présentés à la cour au motif de cette loi $^{39}$. Lorsque la justice pénale est saisie, la preuve est difficile à administrer, aussi cherche-t-on à réparer le préjudice moral subi par la victime, d'où l'introduction dans la constitution de 1988 du crime "pour offense ou délit moral de racisme» ou "pour tort moral». La mutation essentielle, impulsée par le gouvernement consiste alors à criminaliser le racisme. Outre ce renforcement de la législation, l'autre apport majeur de la constitution de 1988 est la restitution par l'Etat des titres respectifs de propriété aux descendants des communautés quilombos, qui désignaient à l'époque esclavagiste les regroupements d'esclaves en fuite. Cette disposition constitutionnelle participe d'une volonté politique de redéfinir l'identité brésilienne. En reconnaissant une communauté dont les pratiques culturelles ont longtemps fait l'objet de discriminations, l'Etat répare publiquement des siècles d'oubli et de négation d'une histoire ignorant le rôle actif de la population noire dans la construction de la nation brésilienne ${ }^{40}$.

Comme en France, ces dispositions légales, constitutionnelles, sont certes des outils indispensables pour lutter contre le racisme mais tendent également à poser l'Etat comme garant d'une société sans discrimination et à le protéger du même coup d'une potentielle responsabilité dans les mécanismes actuels de production du racisme. En effet, on lit au titre premier de la Constitution de 1988 que le Brésil « constitue un Etat démocratique de droit [qui] a pour fondements (...) la dignité de la personne humaine. (...) » (article. premier); «Les objectifs fondamentaux [du Brésil] sont les suivants : I. Construire une société libre, juste et solidaire; (...) III. Eradiquer la pauvreté et la marginalisation et réduire les inégalités sociales et régionales; IV. Promouvoir le bien de tous, sans préjugés d'origine, de race, de sexe, de couleur, d'âge ou toute autre forme de discrimination » (article 3); L'article 5 de la Constitution stipule en outre : «La loi punit toute discrimination portant atteinte aux droits et libertés fondamentaux» (paragraphe XLI); «La pratique du racisme constitue une infraction imprescriptible et pour laquelle il ne sera admis de libération sous caution, elle entraîne une peine de réclusion selon les termes de la loi » (paragraphe XLII) ${ }^{41}$.

Dans les années 1990 on observe un accroissement considérable du nombre de mesures et dispositifs institutionnels pour lutter contre le racisme ${ }^{42}$. A São Paulo, à l'initiative de l'Etat fédéré, un commissariat contre le crime raciste anti-noir et antisémite est créé. La pratique du testing se développe, même si comme en France, le droit ne la reconnaît pas comme preuve. L'Etat met également en place un secrétariat national pour la défense du citoyen qui s'occupe des mesures de lutte contre le racisme, surtout contre les noirs et les juifs ${ }^{43}$.

42 Ces dispositions constituent une avancée de taille en matière de lutte contre le racisme. Néanmoins, reste à savoir si les individus concernés s'en saisissent. Si elles traduisent la remise en question du caractère effectif de la démocratie raciale par les politiques, elles sont souvent présentées en décalage avec l'imaginaire social. Diverses études soulignent en effet les difficultés à reconnaître l'existence du racisme dans la société. La pertinence de la question est sans cesse remise en cause par de multiples enquêtes, 
sondages, qui insistent sur l'indifférence qu'elle suscite, voire le refus de lui accorder de l'importance. France Winddance Twine note ainsi que, pour beaucoup d'Afro-Brésiliens de Vasalia où elle a mené sa recherche, le racisme n'existe pas dans la mesure où la plupart des inégalités raciales ne sont pas perçues ou ressenties comme telles ${ }^{44}$. En conclusion de son étude qui la mène à mettre l'accent sur le désintérêt des individus à l'égard du racisme, elle souligne l'écart entre les mesures prises par l'action antiraciste, les conceptions des chercheurs et celles des membres de la communauté Afrobrésilienne. A partir d'une analyse de la construction des classifications raciales du recensement, Edward E. Telles souligne l'arbitraire et surtout l'instabilité des identifications raciales suivant qu'elles sont établies par les enquêteurs ou les enquêtés ${ }^{45}$.

43 Depuis 1872, excepté durant la période du régime militaire, l'Instituto Brasileiro de Geographia et Estatistica (IBGE) a introduit la dimension raciale dans le recensement de la population. A partir de 1980, on a demandé aux individus d'identifier eux-mêmes leur couleur à partir de trois catégories prédéfinies, à savoir «branco» (blanc), «preto» (noir) et «pardo» (métis) ${ }^{46}$. Telles s'appuie sur le sondage du journal Folha de São Paulo, effectué en 1995, faisant apparaître pour la première fois la réponse de l'enquêteur et de l'enquêté concernant la couleur de la peau, pour montrer que les enquêteurs ont tendance à "blanchir » la population, en particulier sur la base des ressources socio-économiques et du niveau d'études des enquêtés. Selon Robin Sheriff, ce type de pratiques traduit «l'hyper-valorisation » du fait d'être blanc au Brésili" ${ }^{47}$. Rien ne remet en question le fait que les noirs n'aient pas à être «blanchis » dès lors qu'ils réussissent socialement, pas même les déclarations des individus. C'est ainsi que les agents du recensement définissent comme blancs $20 \%$ des individus qui s'identifient pour leur part au groupe métis et disposent en moyenne de revenus supérieurs à ceux qui sont généralement classés dans cette catégorie. Telles souligne l'importance du statut social dans la catégorisation par couleur effectuée par les enquêteurs alors que ces deux variables sont plus fréquemment dissociées par les enquêtés. Ce constat l'amène à démontrer que la classification issue du point de vue des répondants constitue une base de données pertinente pour comprendre les formes de revendication identitaire, alors que celle opérée par les enquêteurs permet de mettre l'accent sur les inégalités raciales. Reste à savoir comment, en sus des interactions entre les deux variables, le racisme est vécu par les individus.

Les chiffres du sondage commandité par le journal Folha de São Paulo en 1995 montrent que si $89 \%$ des Brésiliens pensent qu'il y a du racisme dans la société, seulement $10 \%$ d'entre eux affirment être discriminés ${ }^{48}$. Selon la même étude, $48 \%$ des noirs interviewés, sont d'accord avec l'idée que « les bons noirs ont une âme blanche », ce qui est révélateur des difficultés de la mobilisation contre le racisme. Ce phénomène conduit d'ailleurs une partie du mouvement noir à mettre l'accent sur la nécessité de développer l'éducation contre le racisme. Cependant, comme le souligne Robin Sheriff on a souvent tendance au Brésil à confondre le silence des individus sur la question avec le fait qu'ils n'en ont pas conscience, notamment en raison de l'influence du récit de la démocratie raciale ${ }^{49}$. A travers ses enquêtes, Sheriff montre en effet que le problème concerne moins la reconnaissance de l'existence du racisme que le fait de ne pas en parler. Ce silence socialement partagé - qui implique qu'on ne parle de la question ni dans la sphère privée, ni dans la sphère publique - se comprend par ce qu'elle appelle la censure culturelle, c'est-à-dire une série de comportements, de 
pratiques et de croyances (comme celle de penser que le fait de parler du racisme risque de l'exacerber) qui le rendent non problématique.

Dans quelle mesure peut-on dire que le vécu des discriminations raciales suppose de s'identifier ou d'être considéré comme appartenant à la catégorie "preto", voire «pardo» et dans quelle mesure transcende-t-il cette classification? C'est là qu'une confusion s'installe pour l'émergence de la figure des victimes du racisme. Comme le souligne Angelina Peralva, "le racisme est vécu comme une expérience complexe et multidimensionnelle, avec deux faces principales : celle d'une définition négative du noir (la face raciale) et celle d'une définition négative du favelado (la face sociale) ${ }^{50}$, ces deux définitions étant complémentaires et presque indissociables. Aussi, à partir du moment où le statut social prédomine et influence la désignation raciale des individus, le racisme concerne-t-il uniquement les individus qui sont noirs ou métis et exclus socialement ? Fry rappelle à ce titre que la myriade de catégories de couleur a aussi une fonction. Elle dissipe l'éventuelle polarisation raciale et les tensions. Cette perspective aboutit à définir les formes de discrimination raciale à partir de critères économiques et sociaux et du même coup à limiter le combat contre le racisme à une série de mesures pour lutter contre la pauvreté.

Alors qu'en France le racisme est considéré comme une espèce de virus, de symptôme, provoqué par le chômage et la dégradation des conditions sociales, la lecture raciale de la question sociale au Brésil conduit le plus souvent à pointer les inégalités entre les races comme un état du social plutôt que de les appréhender en tant que discriminations raciales. Autrement dit, la dimension raciale n'est jamais qu'un effet ou une variable et non une cause des problèmes sociaux rencontrés par les individus. Que ce soit en France, où le racisme n'est considéré que comme une conséquence des problèmes sociaux, ou au Brésil où il est sans cesse revu et corrigé par la dimension sociale, le phénomène n'apparaît dans tous les cas qu'en seconde lecture, voire est contourné. Au fond, la reconnaissance du racisme est fondue dans le moule des mythes démocratiques des deux pays. Dans ce cadre, la désignation des victimes du racisme reste floue et instable.

La France admet plus facilement l'existence du racisme mais résiste à prendre en compte les mutations qui s'opèrent autour de l'affirmation des différences. Le Brésil dispose quant à lui de données raciales permettant de faire ressortir les inégalités entre les races mais se concentre essentiellement sur leur caractère social, en grande partie $\mathrm{du}$ fait des systèmes de conversion qui s'opèrent entre la couleur de la peau et le statut social. Le métissage offre certes une possibilité de jouer avec les catégories raciales, mais la polarisation raciale - et les présupposés biologiques sur lesquels elle repose place cette capacité sous contrainte ${ }^{51}$. Par ailleurs, les références au métissage ne servent pas les mêmes interprétations. Elles peuvent être en effet tout autant au service d'une idéologie qui justifie certaines formes de domination sociale dans les couches sociales supérieures que la revendication implicite d'une commune humanité. La dénonciation du racisme n'aboutit pas toujours à une forme de politisation du problème. Au Brésil la tension majeure semble se situer entre la logique de criminalisation du racisme et le manque de mobilisation des Afro-brésiliens. En France, la tension se joue entre la logique d'intégration des populations immigrées et de leurs descendants et la logique de dénonciation du racisme.

Vers de nouvelles formes de reconnaissance : les mythes en débat 

fait que l'expérience vécue de la victime représente un enjeu fondamental pour l'expression et la reconnaissance de la discrimination raciale. Dans de nombreux pays, divers dispositifs institutionnels encouragent en effet les victimes à faire des signalements afin d'améliorer concrètement leur situation. De même, les études portant sur le racisme et les discriminations s'intéressent de plus en plus au point de vue des acteurs directement concernés. Désormais, le racisme est appréhendé comme un processus produit de façon routinière et renforcé à travers les pratiques du quotidien. C'est dans cette perspective qu'une partie des recherches appréhende le phénomène en termes de everyday racism (racisme au quotidien) en s'intéressant à la façon dont les individus perçoivent, vivent et combattent les discriminations raciales.

années 1990, un tournant s'opère dans la littérature anglo-américaine sur le racisme dont le sujet central se réoriente sur la question du vécu de la discrimination raciale. Comme le soulignent Joe Feagin et Melvin Sikes, «le racisme moderne doit être interprété comme une expérience vécue (...) Les expériences discriminantes sont non seulement douloureuses et angoissantes immédiatement et dans leurs conséquences mais ont également un impact cumulatif sur des individus particuliers, leur famille et leur communauté (...) L'impact cumulatif de situations d'hostilité raciale répétées est plus dommageable que la somme de ces expériences peut en avoir l'air pour l'observateur habitué (...) L'expérience répétée de formes de racisme affecte de façon significative le comportement et l'approche de l'existence d'une personne noire ${ }^{52}$. besoin de reconnaissance. Dès lors que la situation socio-économique des individus ne détermine pas tout, comment trouver des politiques qui permettent d'assurer la reconnaissance du racisme dans une société autrement qu'en jouant sur ses sources sociales ? C'est à partir de cette question que les interrogations sur les cadres normatifs des deux pays apparaissent progressivement.

De l'intégration socio-économique comme réponse au racisme à l'intégration culturelle comme exigence de reconnaissance des discriminations raciales

51 Jusqu'à la fin des années 1990, les mesures pour combattre le racisme en France ne s'inscrivent que par touches dans l'agenda public qui reste dominé par le thème phare de l'intégration. En 1989, naît le Haut conseil à l'Intégration dont les travaux portent essentiellement sur les dangers des tendances au communautarisme. En 1991, des cellules départementales de coordination de la lutte contre le racisme sont expérimentées dans quelques départements pour être ensuite généralisées dans le cadre des conseils départementaux de prévention de la délinquance à partir de 1993. Ce projet invitait en fait les préfets à mettre au moins une fois par an la lutte contre le racisme à l'ordre du jour de ce conseil, ce qui montre la marginalité du sujet comparativement à la permanence du thème des violences urbaines dans les débats politiques et les médias ${ }^{53}$. Toujours est-il que la figure de la victime du racisme se retrouve la plupart du temps en balance avec celle du délinquant ou celle du refus de l'intégration culturelle (lorsqu'elles ne se confondent pas d'ailleurs). L'image des jeunes de banlieues, envahie par l'association entre immigration et délinquance tend à déformer et à s'éloigner de la réalité vécue des discriminations raciales ${ }^{54}$. A partir de cette articulation, les politiques publiques ont surtout remis en cause l'intégration culturelle des populations immigrées et de leur descendance en leur faisant porter la charge du projet d'intégration. C'est dans ce cadre que l'on a parlé à partir du milieu 
des années 1980 dans le discours savant comme dans les médias et dans le discours politique, de l'idée et de l'expression d'une « crise du modèle d'intégration ».

De façon générale, ce n'est pas tant sur les figures des victimes que sur celles des auteurs du racisme que se fixe l'attention. Au fil des élections, les scores en progression du FN conduisent le débat à se polariser sur cet enjeu tout au long des années 1990. La poussée de l'extrême droite a participé de la crispation, voire du refus de la société à voir la diversité des cultures et des identités qui y sont présentes et en perpétuelle transformation. Les premiers grands débats publics sur le voile qui apparaissent en 1989 autour des jeunes lycéennes de Creil font émerger des versions caricaturales et intransigeantes du modèle républicain d'intégration. L'association SOS racisme qui s'oppose à l'exclusion des jeunes filles est alors accusée de défendre «le droit à la différence ». Cette interprétation par les médias et de nombreux politiques, intellectuels, de gauche comme de droite, déclenche une polémique sur l'antiracisme, alors en grande partie associé au nom de SOS racisme, qui remet en cause l'efficacité de son action $^{55}$. En se prononçant contre la guerre du Golfe, l'association perd de nombreux soutiens ${ }^{56}$.

Tout au long des années 1990, le racisme est essentiellement rapporté aux phénomènes que condense l'idée d'exclusion s'imposant désormais dans les débats intellectuels et politiques. Le constat central à l'origine de cette articulation consiste à dire que l'exclusion surtout sociale produit le racisme. A cet égard, les quartiers populaires des banlieues où l'immigration est fortement représentée sont perçus comme le berceau du racisme qui serait la manifestation du sentiment d'abandon, de désarroi des populations les plus démunies. Phénomène que l'enquête de Michel Wieviorka sur la France raciste, menée au début des années 1990 a largement nuancé en montrant que le racisme s'associe moins aux situations d'exclusion sociale qu'à de grandes inquiétudes culturelles ainsi qu'à des peurs identitaires ${ }^{57}$.

54 A la fin des années 1990, cette perspective se renforce à travers diverses approches qui mettent l'accent sur la production du racisme au cœur de la société. Du côté des études en sciences sociales, l'enquête menée par Philippe Bataille sur le monde du travail a notamment révélé que les discriminations raciales touchent l'ensemble de la société et se traduisent par des pratiques, voire des mécanismes institutionnalisés qui ont cours dans le cadre de l'organisation du travail ${ }^{58}$. Sur le plan associatif, plusieurs initiatives témoignent d'un volontarisme à prendre sur le fait les comportements discriminatoires. Les campagnes de testing ${ }^{59}$, organisées par SOS racisme depuis 1999, témoignent d'une action plus engagée auprès des victimes et des obstacles qu'elles rencontrent dans divers domaines (emploi, logement, loisirs). En 2001, le MRAP a ouvert près de cinquante permanences d'accueil pour encourager les victimes et les témoins de discrimination à réagir et à entamer des démarches ${ }^{60}$. Propos racistes proférés au cours d'un différend entre voisins, lors d'un entretien d'embauche, entre collègues, refus d'ouverture d'un compte bancaire, refus d'embauche, doute ou certitude d'être discriminé, autant de cas qui marquent l'étendue et la banalité de ces phénomènes au quotidien.

Cette lecture du racisme s'appuyant sur la réalité des discriminations raciales, c'est-àdire le traitement défavorable d'une personne ou d'un groupe en raison de son origine réelle ou supposée, de son apparence ou encore son patronyme, élargit considérablement la reconnaissance du phénomène qui dépasse désormais le cadre des quartiers défavorisés ainsi que le champ de ses formes les plus flagrantes telles l'insulte 
ou la violence physique. C'est dans ce contexte que l'action publique, largement influencée par l'agenda de l'Union européenne ${ }^{61}$ a déployé une politique spécifique de lutte contre les discriminations raciales avec la volonté de «déplacer l'accent d'une réflexion sur les carences des candidats à l'intégration vers une réflexion sur les raideurs de la société d'accueil $»^{62}$. Autrement dit, d'une politique exclusivement centrée sur l'intégration, c'est-à-dire sur le passage de « l'extérieur » vers "l'intérieur » de la société, le débat se déplace sur le fonctionnement même de la société ${ }^{63}$.

L'aide aux victimes devient alors un des axes majeurs du programme de lutte contre les discriminations. Un groupe d'étude (le Groupe d'Etudes et de Lutte contre les Discriminations, GELD) et un dispositif sont prévus à cet effet. En 1999 sont créées les Commissions Départementales d'Accès à la Citoyenneté (CODAC). Implantées dans chaque préfecture et composées de référents à la fois institutionnels et associatifs, leur mission consiste dans un premier temps à inscrire les problèmes spécifiques rencontrés par les jeunes issus de l'immigration et plus généralement par l'ensemble des personnes issues des quartiers en difficulté dans les grandes politiques contractuelles de l'Etat, tels que les emplois jeunes. En devenant par la suite le relais départemental du 114, plate-forme téléphonique nationale gratuite, mise en place en mai 2000, les commissions départementales deviennent "un lieu d'écoute, de réflexion, d'impulsion et de mise en cuvre des actions destinées à lutter contre les discriminations ${ }^{64}$.

C'est dans ce cadre qu'une mutation majeure s'opère dans le combat contre le racisme. D'un rôle encore très imprégné du thème de l'intégration et axé sur des actions de sensibilisation, on passe en effet ici à une implication au plus près des individus concernés. Le dispositif d'aide directe aux victimes ouvre la voie à une forme inédite de reconnaissance individuelle du racisme par les autorités publiques. Concrètement, les écoutants du numéro vert 114 sont chargés de remplir des fiches d'appel permettant d'identifier l'appelant et de rapporter les faits discriminants afin de les transmettre à la CODAC la plus proche. Sur place, un ou plusieurs référents sont censés orienter et accompagner les victimes dans leurs démarches.

Le développement de la discrimination positive au Brésil : prolongement ou contradiction de la démocratie raciale?

Jusqu'au milieu des années 1990 au Brésil, rares sont les voix qui s'élèvent pour dénoncer le racisme en tant qu'élément structurant certains rapports sociaux et pratiques institutionnelles. Comme le souligne Sergio Adorno, seules quelques études ont en effet démontré les logiques discriminantes portées par les institutions. A travers son analyse du système pénal, il montre par exemple dans quelle mesure la couleur de peau est un facteur déterminant dans les différences de traitement des individus au sein du système judiciaire. Il apparaît notamment que $58 \%$ des accusés noirs ont été arrêtés en flagrant délit contre $46 \%$ d'accusés blancs. Par ailleurs, $62 \%$ des noirs sont défendus par un avocat commis d'office contre $39,5 \%$ des blancs ${ }^{65}$.

Vers le milieu des années 1990, apparaissent progressivement des campagnes de sensibilisation pour lutter contre les manifestations quotidiennes du racisme et des discriminations raciales. Dans les immeubles et les résidences de haut standing, on dénonce par exemple le fait que les noirs sont censés n'utiliser que les entrées et les ascenseurs de service. Comme le met en scène une bande dessinée du "Conselho de Desenvolvimento da Comunidade Negra » de l'Etat de Bahia ${ }^{66}$, les noirs se voient parfois interdire l'entrée de lieux publics comme les boîtes de nuit à la mode, et plus communément les supermarchés où ils sont fréquemment soupçonnés de vol. Un noir 
aperçu de nuit dans un quartier résidentiel par une patrouille de police est immédiatement interpellé pour vérification d'identité et interrogé sur le motif de sa présence en ce lieu, les noirs n'étant supposés résider que dans les favelas et à la périphérie des villes.

Pendant que les politiques publiques françaises déploient une logique défensive pour lutter contre les discriminations, le Brésil développe une logique contre-offensive à travers des mesures de discrimination positive. En 1995, le gouvernement prend une série de mesures en faveur des noirs et des métis. Il s'agit à la fois de valoriser cette population en reconnaissant le rôle joué par les noirs dans la construction matérielle et culturelle du Brésil, et de mettre en œuvre des politiques propres à garantir à tous l'égalité d'accès aux avantages de la citoyenneté. Le 20 novembre 1995 est choisi comme "Jour de la conscience noire ", en hommage à Zumbi, chef noir du célèbre quilombo de Palmarès, assassiné par les autorités coloniales en 1695, élevé au rang de héros national. Dans le même temps, un groupe de travail interministériel est mis en place afin d'assurer la représentation de la population noire dans l'emploi, les médias (promotion d'une image appropriée des noirs dans les médias et les programmes culturels officiels), l'éducation (promotion de la non-discrimination et de la tolérance dans le système scolaire, choix de manuels mettant en valeur, sans stéréotype ni discrimination, l'histoire et les combats du peuple noir dans la construction de la nation brésilienne), ou encore la santé ${ }^{67}$.

61 Ces mesures gouvernementales renforcent l'action des associations, en particulier de femmes, qui luttent depuis de nombreuses années contre le racisme. Il s'agit notamment d'associations telles que Gélédès, née en 1988, et Fala Preta, créée en 1997, qui articulent le traitement de formes diverses de discrimination. Leurs initiatives permettent de mêler, au sein de la même structure, la prise en charge d'oppressions et de discriminations relevant de différents champs (violence domestique, racisme, problèmes de santé). Cette perspective prend en compte l'accumulation, voire l'expérience simultanée de plusieurs types de discriminations par un même individu ce qui lui laisse une latitude d'action plus grande pour se défendre.

62 Le travail de ces associations traduit par ailleurs un changement important des organisations liées au mouvement noir. Auparavant, ces dernières se concentraient essentiellement sur l'élaboration d'une identité noire spécifique. Or, si la construction d'une identité raciale est toujours importante, il existe un intérêt grandissant pour les inégalités dans le domaine de l'emploi, de l'éducation ou encore de la santé.

Depuis le milieu des années 1990, le Brésil n'a cessé de renforcer son dispositif de discrimination positive dans le domaine de l'éducation. Il a ainsi été décidé que $10 \%$ des places dans les établissements d'enseignement supérieur seraient réservées aux jeunes appartenant à des groupes victimes de discrimination. Par ailleurs, les programmes d'enseignement de l'Etat de São Paulo contiennent désormais une section qui traite de la question du racisme. De même, les programmes d'études scolaires et universitaires du pays ont intégré une matière intitulée "Histoire et culture de l'Afrique", permettant de souligner la place qui revient aux cultures africaines dans l'édification de la société brésilienne. Il existe désormais au Brésil un système selon lequel $40 \%$ des personnes qui participent aux activités réalisées par les médias pour le compte de l'Etat doivent être de couleur. L'objectif est également de montrer dans les médias l'importance du rôle des noirs dans l'édification de la société brésilienne ${ }^{68}$. 
64 Au niveau international, la participation du Brésil au projet non gouvernemental auquel plusieurs autorités brésiliennes ont participé - Beyond racism, en collaboration avec les Etats-Unis et l'Afrique du Sud, affiche la volonté de promouvoir la reconnaissance $\mathrm{du}$ racisme et des discriminations raciales ${ }^{69}$. Cette initiative a notamment donné lieu à un travail statistique qui révèle, domaine par domaine, le poids de la variable raciale sur les inégalités entre individus. On apprend par exemple qu'environ $26 \%$ des noirs, comparativement à $16 \%$ des blancs, gagnent moins que le revenu minimum, alors que $1 \%$ des noirs touchent 10 fois le revenu minimum contre $4 \%$ de blancs. Deux tiers des enfants africains brésiliens (African Brazilian) ont accès à l'éducation de base comparativement à $85 \%$ des enfants blancs. On enregistre des écarts tout aussi flagrants concernant le chômage, la santé, l'espérance de vie, la représentation médiatique, qui reflètent clairement les inégalités touchant la population noire.

65 De même, la conférence mondiale contre le racisme qui s'est tenue à Durban en 2001 a donné une nouvelle impulsion aux politiques de discrimination positive. En réponse aux attentes formulées lors de cet évènement, le ministre du Développement agricole a annoncé un « Programme de discrimination positive pour les hommes et les femmes noirs ». Cette action vise notamment à réduire les inégalités entre les fonctionnaires et les bénéficiaires de la réforme agricole et de l'agriculture familiale. Cela implique entre autres la mise en place de quotas pour la participation des hommes et des femmes noires aux positions administratives. Par ailleurs, l'assemblée législative de Rio de Janeiro a annoncé que $40 \%$ des entrées à l'université seront réservées aux noirs et aux métis ${ }^{70}$.

66 Plus récemment, un bureau du Secrétaire spécial pour la promotion de l'égalité raciale est chargé depuis mai 2003 de coordonner les activités menées par le Gouvernement pour combattre la discrimination raciale et promouvoir l'égalité raciale, y compris en matière d'emploi, de culture, d'éducation et de santé. Cette instance a précisé que la politique de discrimination positive prenait en compte les personnes d'ascendance africaine et a fait état de la nomination du premier juge noir à la Cour suprême.

67 La mise en œuvre de politiques de discrimination positive au Brésil donne une nouvelle épaisseur au débat sur la question raciale. Après avoir misé sur une logique répressive et défensive pour endiguer le racisme, l'Etat brésilien en faisant preuve d'un volontarisme politique pour corriger directement les effets des discriminations raciales reconnaît une forme de responsabilité dans leurs mécanismes de production. En veillant directement à la représentation et l'intégration des populations noires et métisses dans les institutions, il souligne en effet que l'absence de ces politiques participe des mécanismes discriminants à l'œuvre dans la société.

68 Cette logique contre-offensive a déclenché une polémique sur la remise en question qu'elle porte de la démocratie raciale. Pour César Benjamin, la discrimination positive est une espèce de non-sens au vu du continuum de couleur de la population brésilienne. Selon lui, «constituer une identité basée sur la race est particulièrement réactionnaire $»^{71}$. Ainsi, une politique de quotas en faveur des noirs constitue un anachronisme. Le principal argument des détracteurs de la discrimination positive repose ainsi sur le danger de l'usage d'une catégorie biologique par les politiques publiques au détriment de l'idée de démocratie raciale. En d'autres termes, les quotas redonneraient une vitalité à la notion de race que le récit de la démocratie raciale avait su fondre dans un mythe, supposé plus effectif qu'il ne l'est cependant. Peter Fry va un 
peu dans le même sens en soulignant que même si le mythe de la démocratie raciale semble constamment désamorcer la conscience des discriminations raciales et du racisme, il est crucial de dissocier la volonté de minimiser la pertinence de l'idée de race dans la vie sociale (en combattant le racisme sur le lieu de travail, à l'école, dans les cours de justice) de celle d'élaborer des politiques qui se fondent sur l'idée de race comme critère pour la distribution des ressources publiques. Selon lui, le discours de l'affirmative action revient à reconnaître l'existence des races. Sueli Carneiro, présidente de l'association Gélédès insiste quant à elle sur l'impératif que représentent ces politiques dans un pays où le noir ne peut être réduit à une réalité statistique et laissé en attente d'une ascension sociale improbable. Elle rappelle à cet égard que seuls $2 \%$ de la population noire a accès à l'université ${ }^{72}$.

69 La nécessité de la discrimination positive révèle une part des leurres de la démocratie raciale. Le Brésil a certes déconstruit le tabou autour de la question raciale qui atténue largement l'image édénique d'harmonie raciale qu'il affichait ${ }^{73}$. Reste à savoir comment le pays peut trouver un modus vivendi pour appliquer cette politique sur la base du continuum de couleur de sa population.

70 Tout en développant une logique de répression des actes et des comportements racistes, le Brésil et la France ont longtemps résisté à prendre à bras le corps la question du racisme. Avant d'être reconnu comme une expérience vécue, comme une atteinte à l'identité de personnes singulières ou de groupes, le phénomène a surtout été appréhendé dans ces deux pays à partir de ses mécanismes de production, des sources sociales susceptibles de l'alimenter, ce qui a souvent conduit à en négliger les conséquences dans la vie des individus. Cette perspective a non seulement contourné la responsabilité de l'Etat, mais elle a également privé la lutte contre le racisme d'actions politiques concrètes s'attaquant de front au phénomène.

71 La seconde moitié des années 1990 marque cependant un tournant dans les deux pays qui développent des politiques d'aide directe aux victimes. Au Brésil comme en France, ces processus relèvent plus de l'action de l'Etat que de la mobilisation des populations victimes qui se reconnaissent plus ou moins dans les dispositifs mis en place.

72 La France a développé ces dernières années un dispositif institutionnel chargé de recueillir les plaintes des victimes. La prise en compte de la dimension raciale, ethnique ou culturelle mise en mot par les individus a bouleversé et dépassé les définitions habituelles du racisme souvent fondées sur les discours et les arguments racistes. Le principal défi que soulèvent ces récits dans le cadre d'un traitement institutionnel personnalisé des discriminations raciales est l'articulation qui se joue entre leurs conceptions objectives et subjectives, autrement dit entre leurs représentations collectives, et leur vécu. A cet égard, le mérite d'un dispositif tel que le numéro d'appel gratuit 114 a été de mettre en lumière les lignes de tensions entre ces deux éléments qui ont souvent du mal à s'articuler dans le cadre des institutions ${ }^{74}$. Cet élément montre que l'enjeu de la lutte contre le racisme n'est pas de renforcer les sanctions mais surtout de pouvoir les appliquer. La transmission des plaintes au ministère public demeure rare, en partie du fait de la décision des institutions en question (police, justice) à juger inutile de traiter le cas, mais également du fait de la réticence des individus à entamer une procédure longue, coûteuse et jugée parfois trop lourde au regard des attentes des victimes. La création récente de la Haute autorité de lutte contre les discriminations et pour l'égalité (HALDE) marque un nouveau tournant dans le processus d'institutionnalisation de la lutte contre le racisme ${ }^{75}$. 

positive $^{76}$. Cette décision donne une visibilité accrue à la problématique raciale, endormie par la force du récit de la démocratie raciale et pose la question centrale de la désignation et du repérage des individus à qui s'adresse cette mesure.

La multiplication et le renforcement des affirmations culturelles, ethniques, religieuses observables au sein de ces sociétés plaident également pour une nouvelle approche du racisme. Au Brésil, cette tendance procède de la poussée d'une identité afro-brésilienne qui force à revoir le constat longtemps prégnant du manque, voire de l'absence de mobilisation des Afro-brésiliens ${ }^{77}$. Dès lors, comment vont s'articuler les logiques de développement de la discrimination positive et celles de l'affirmation identitaire? En France, une partie des dénonciations actuelles par lesquelles transitent l'affirmation des identités post-coloniales ainsi que les revendications construites autour de la mémoire de la traite et de l'esclavage lancent un défi aux politiques de reconnaissance et ouvrent un immense chantier pour reconsidérer le rapport entre le racisme vécu au présent et au passé. Les variantes du phénomène ne sont pas seulement à repenser à la lumière de l'histoire, de la mémoire, mais également du point de vue de la dimension spatiale qu'elles mettent en jeu. Dans quelle mesure doivent-elles être pensées dans les frontières de l'Etat nation? Se positionner en tant que victime, ou se reconnaitre dans la haine de tel ou tel groupe (voire les deux) peut se nourrir de l'identification à des vies, des expériences - du moins à l'idée qu'on s'en fait - loin des frontières du pays dans lequel on vit. A plusieurs reprises, le retour de l'antisémitisme ${ }^{78}$ dans la société française a été rapporté aux formes d'identifications et aux opinions liées au conflit israélo-palestinien. Ainsi, dans quelle mesure le racisme est-il influencé, inspiré mais aussi détourné ou réinterprété par des logiques globales?

Les tentatives pour relier les dimensions objectives et subjectives de la lutte contre le racisme et les discriminations raciales transforment l'espace de conflits pour la reconnaissance de ces phénomènes. Les politiques d'aide directe aux victimes que l'on observe en France et au Brésil - qu'elles prennent la forme d'un dispositif de traitement des plaintes ou celle d'une politique de discrimination positive - produisent, révèlent et mettent en circulation de multiples versions du fait d'être discriminé qui parfois se télescopent, s'opposent ou encore rentrent en concurrence. Cette mutation lance de nouveaux défis au traitement du racisme dans ces deux pays tel celui de penser ensemble différents types d'expérience vécue du racisme et d'y apporter des réponses. La nécessité d'identifier les victimes - ce dont la lutte contre le racisme se passait lorsqu'elle se cantonnait à une condamnation morale - en bouleversant les logiques d'appartenance à un groupe, affaiblit en effet la conception unifiée du combat contre le racisme et soulève la question des articulations entre le registre de la dénonciation et celui de l'affirmation des différences. 


\section{NOTES}

1.. Boli J., Thomas G., « World Culture in the World Polity : A Century of International Non-Governmental Organisation », American Sociological Review, n62, 1997, pp. 171-190. 2.. Les délégués de la conférence ont par exemple refusé l'accréditation d'une organisation non-gouvernementale, l'Association internationale des homosexuels et lesbiennes, alors que l'Union européenne et d'autres pays ont défendu sa participation au nom de la non-discrimination. « La Conférence mondiale contre le racisme est mal partie ", Le Monde, 4 août 2001.

3.. Ce thème est central dans l'analyse des spécialistes du racisme mais également dans la littérature brésilienne. L'oeuvre du dramaturge Nelson Rodrigues est marquée par cette question et dénonce ce qui se cache derrière les apparences en mettant le doigt sur une farce dont les Brésiliens s'enorgueillissent bien souvent - à savoir, la croyance qu'au Brésil, on vit dans une démocratie raciale. Rodrigues N., L'ange noir, Paris, Collection des Quatre-vents, 1988 (1947).

4.. Comme l'indique le dernier recensement de l'IBGE (les noirs sont ceux qui s'autodéclarent noirs ou « foncés »). Voir le lien suivant : www.ibge.gov.br.

5.. Park R.E., Race and Culture, New York, The Free Press, 1950.

6.. Ibid, p. 81.

7.. Pour autant, il ne s'agit pas d'introduire ou de défendre une opposition entre application des principes républicains et ethnicisation ou reconnaissance des différences. Catherine Rassiguier révèle une image plus problématique et nuancée du modèle français d'intégration en mettant en lumière les contradictions concernant le traitement de la femme immigrée dans l'histoire du code de la nationalité. Cette auteure montre le mouvement de fermeture des frontières nationales et l'ethnicisation des membres nationaux qu'ont inspiré les vagues d'immigration d'après-guerre et postcolonialistes, ainsi que la crise économique des années 1970 en Europe. A travers les changements dans le code de la nationalité en 1927, 1945 et 1973 qui affectent les femmes en général et les femmes étrangères en particulier, Rassiguier montre que les processus d'exclusion et d'ethnicisation ont changé sur le temps mais loin d'être des anomalies au sein de la tradition républicaine, ils sont en fait constitutifs de cette tradition. Voir Rassiguier C., « Gender, Race and Exclusion : A New look at the French Republican Tradition », International Feminist Journal of Politics, vol.1, n³, 1999, pp. 435-457.

8.. Bennassar B., Marin R., Histoire du Brésil, 1500-2000, Paris, Fayard, 2000.

9.. Nogueira O., Tanto Preto Quanto Branco: Estudos de Relações Raciais, São Paulo, T.A. Queiroz, 1985.

10.. Azevedo C.M.M. de, «Abolicionismo e memória das relações raciais », Estudos AfroAsiáticos, 26, 1994, pp. 5-20.

11.. Maio M.C., «O Projeto Unesco e A Agenda das Ciências Sociais no Brasil dos Anos 40 e 50 ", Revista Brasileira de Ciências Sociais, vol. 14, n 41, 1999, pp. 141-158.

12.. Pereira de Queiroz M.I., « Identidade cultural, identidade nacional no Brasil, Tempo Social », Revista de Sociologia da USP, vol. 1, n¹, 1989.

13.. Andrade M. de, Macounaima, Paris, Stock/Unesco/Allca XX, 1996 (1928).

14.. Freyre G., Casa Grande e Senzala : formaçao da Familia Brasileira sob o regime da economia patriarcal, Rio de Janeiro, Maia \& Schmidt, 1933. 
15.. Guimarães A.-S.-A., « Démocratie raciale », Cahiers du Brésil Contemporain, Paris, n49-50, 2002, pp. 11-38.

16.. Bastide R., Fernandes F., Relaçoes Raciais entre Negros e Brancos em São Paulo, São Paulo, Unesco-Anhembi, 1955.

17.. Voir notamment le livre de Zweig S., Le Brésil, terre d'avenir, Paris, L'Aube, 1992, (1941).

18.. Pierson D., Negroes in Brazil: A Study of Race Contact in Brazil, Carbondale, Southern Illinois University Press, 1942.

19.. Bastide R., « Les relations raciales au Brésil », Bulletin International des Sciences Sociales, Unesco, vol. 9, n4, 1957, pp. 525-543.

20.. Ibid., p. 536.

21.. Fernandes F., A Integração do Negro na Sociedade de Classes, 3 ème édition, São Paulo, Atica, 1978 ; Fernandes F., The Negro in Brazilian Society, New York, Columbia University Press, 1969.

22.. Bradford Burns E., A History of Brazil, $3^{\text {ème }}$ édition, New York, Columbia University Press, 1993.

23.. Gastaut Y., « Le racisme anti-maghrébin et les séquelles de la guerre d'Algérie », Hommes et Migrations, $n^{\circ} 1174$, mars 1994, pp. 35-42.

24.. Weber J., « Racisme pas d'âge », L'humanité, 17 octobre 1978.

25.. Stora B., Le transfert d'une mémoire. De «l'Algérie française » au racisme anti-arabe, Paris, La découverte, 1999, p. 91.

26.. Par l'ordonnance du 29 décembre 1958, le FAS est défini comme un fonds destiné à promouvoir une action sociale et familiale en faveur des salariés travaillant en France métropolitaine dans les professions visées par le régime algérien d'allocations familiales et dont les enfants résident en Algérie.

27.. Gastaut Y., « 'Sommes nous racistes ?’ Evolution d'une question angoissante dans l'espace public français ", Migrations Société, vol. 9, n49, janvier-février 1997, pp. 53-66. 28. Gastaut Y., « La flambée raciste de 1973 en France », Revue Européenne des Migrations Internationales, vol. 9, $\mathrm{n}^{\circ} 2,1993, \mathrm{pp} .61-75$.

29.. Gastaut Y., « Le racisme anti-maghrébin et les séquelles de la guerre d'Algérie », Hommes et Migrations, $n^{\circ} 1174$, mars 1994, pp. 35-42.

30.. Weil P., La France et ses étrangers, l'aventure d'une politique de l'immigration, 1938-1991, Paris, Calmann-Lévy, 1991.

31.. Harlem Désir interviewé par Pierre-Yves Le Priol, « Gaffe aux récupérations », La Croix, 15 juin 1985, déclare : "Je pense qu'il existe un vrai problème du racisme en France et qu'il ne suffit pas pour le combattre de crier : 'égalité des droits' à tout bout de champ. On ne peut pas abolir ce racisme-là par décret ni par simple mesure égalitaire. Moi je crois plutôt à un travail à faire dans les mentalités. Voyez les Antillais ou les jeunes 'beurs' français : ils ont théoriquement les mêmes droits que les métropolitains et pourtant ils souffrent du racisme ». 32.. Dubet F., La Galère : jeunes en survie, Paris, Fayard, 1987.

33.. Dubet F., Lapeyronnie D., Les quartiers d'exil, Paris, Seuil, 1992.

34.. Hasenbalg C., Discriminaçao e desigaldades no Brasil, Rio de Janeiro, Graal, 1979 ;

Nelson do Valle S., « Updating the cost of not being white », in Fontaine M. (dir.), Race, class and power in Brazil, Los Angeles, University of California Press, 1985, pp. 42-55.

35.. Guimarães A.-S.-A., «Intelectuais negros e formas de integração nacional », Estudos Avançados, vol. 18, n50, 2004, pp. 271-284.

36.. Peralva A., Violence et démocratie. Le paradoxe brésilien, Paris, Balland, 2001, p. 33. 
37.. Hanchard M.-G., Orpheus and Power : the movimento negro of Rio de Janeiro and São Paulo, Brazil, 1945-1988, Princeton, Princeton University Press, 1994 ; Skidmore T.-E., «Fact and Myth : discovering a racial problem in Brazil, São Paulo », Instituto de Estudos Avançados, 1992 ; Telles E.-E., « Ethnic boundaries and political mobilisation among African Brazilians : comparisons with the US case », in Hanchard M. (dir.), Racial Politics in Contemporary Brazil, London, Duke University Press, 1999 ; Winant H., « Rethinking Race in Brazil », Journal of Latin American Studies, n²4, 1992, pp. 173-192 ; Burdick J., « The lost constituency of Brazil's black movements », Latin American Perspectives, 25 (1), 1998, pp. 136-155.

38. Voir les travaux d'Agier, Sansone ou Guimarães qui analysent l'émergence d'un « monde noir » à travers la redécouverte et la valorisation des « racines » africaines, l'ethnicisation des pratiques culturelles ou la construction d'une territorialité afrobrésilienne. Ces auteurs soulignent cependant le caractère partiel, localisé et fragmenté de ces expressions, peinant à se structurer en un mouvement politique pérenne au cours des années 1990. Voir Agier M., « Ethnopolitique. Racisme, statuts et mouvement noir à Bahia ", Cahiers d'Etudes Africaines, n¹25, 1992, pp. 53-81; Sansone L., From Africa to Afro, Use and Abuse of Africa in Brazil, the South-South Exchange Programme for Research on the History of Development (SEPHIS) and the Council for the Development of Social Science Research in Africa (CODESRIA), Amsterdam/Dakar, 1999 ; Guimarães A.-S.-A., Racismo e Anti-racismo no Brasil, São Paulo, Editora 34, 1999.

39.. Eccles P., Culpados até prova em contrário : os negros, a lei e os direitos humanos no Brasil, Estudos Afro-asiaticos, 1991.

40.. Véran J.-F., « Brésil : les découvertes du « quilombo ». La construction hétérogène d'une question nationale ", Problèmes d'Amérique Latine, n³2, 1999.

41.. Edition mise à jour en 1994 [texte du 5 octobre 1988 modifié par les amendements $n^{\circ} 1 / 92$ à $4 / 93$ et $n^{\circ} 1 / 94$ à 6/94], traduction française de J. Villemain, mise à jour de J.F. Cleaver, Brasilia, Sénat fédéral, 1994.

42.. Guimarães A.-S.-A., Racial Inequalities, Black Protest and Public Policies in Brazil, Paper prepared for the United Nations Research Institute for Social Development (UNRISD) Conference on Racism and Public Policy, septembre 2001, Durban, Afrique du Sud. 43.. Ces informations sont tirées du rapport de Maurice Glèlè-Ahanhanzo, rapporteur spécial sur les formes contemporaines de racisme, de discrimination raciale, de xénophobie et de l'intolérance qui y est associée, sur la mission qu'il a effectuée au Brésil du 6 au 17 juin 1995, conformément aux résolutions 1993/20 et 1995/12 de la commission des droits de l'Homme, Conseil économique et social des Nations Unis, 23 janvier 1995.

44.. Winddance Twine F., " Mapping the terrain of Brazilian racism », Race and Class, vol. 38, n³, 1997, pp. 49-61.

45.. Telles E.-E., Lim N., « Does it Matter Who Answers the Race Question?: Racial Classification and Income Inequality in Brazil », Demography, 35(4), 1998, pp. 465-474. Voir également du même auteur, Racismo à brasileira: uma nova perspectiva sociológica, Rio de Janeiro, Relume-Dumará, Fundação Ford, 2003.

46.. Nobles M., Shades of Citizenship: Race and the Census in Modern Politics, Stanford, Stanford University Press, 2000.

47.. Sheriff R., Dreaming Equality: Color, Race, and Racism in Urban Brazil, New Brunswick, Rutgers University Press, 2001, p. 142. 
48.. Des disparités importantes sont cependant à noter suivant les régions. $17 \%$ des personnes interviewées dans le nord-est affirment subir des discriminations en raison de leur couleur, contre $8 \%$ dans le sud-est.

49.. Sheriff R., " Exposing Silence as Cultural Censorship : A Brazilian Case », American Anthropologist, vol. 102, $\mathrm{n}^{\circ} 1,2000$, pp. 114-132.

50.. Peralva A., Violence et démocratie. Le paradoxe brésilien, op. cit., p. 53.

51.. Agier M., Anthropologie du carnaval. La ville, la fête et l'Afrique à Bahia, Marseille, éditions Parenthèses/IRD, 2000, p. 228.

52.. Feagin J., Sikes M., Living with racism, Boston, Beacon, 1994, pp. 15-17.

53.. Peralva A., Macé E., Médias et violences urbaines. Débats politiques et construction journalistique, Paris, La Documentation française, 2002.

54.. "Les immigrés n'apparaissent à la une des journaux qu'en cas de malheur (...). Petit à petit s'opère une espèce de transfert dans l'esprit des gens entre 'immigré' et 'problème' (...). Les médias ont probablement involontairement participé à instaurer un immense malentendu entre les immigrés et la France (...), mais (...) l'intégration se fait dans la société, et plus elle devient irréversible, plus la résistance de ceux qui refusent le réel est violente. Ce qui fait peur, ce n'est la différence, c'est la ressemblance. Et cela est rarement présent dans les médias ", in M'scope, «Image de l'immigration dans les médias », dossier, 1993, pp.33-44. Pour une lecture critique de l'accumulation des griefs fait au garçon arabe de banlieue, voir également Guénif N. et Macé E., Les féministes et le garçon arabe, Paris, L’Aube, 2004.

55.. De Closets F., La Grande Manip, Paris, Le Seuil, 1990. Ce livre met en cause le discours moral de SOS-racisme, accusé de produire des clivages idéologiques plutôt que d'aider à résoudre les problèmes concrets.

56.. Juhem P., SOS-racisme, histoire d'une mobilisation " apolitique ». Contribution à une analyse des transformations des représentations politiques après 1981, Thèse de doctorat (Dir : Bernard Lacroix), Paris X Nanterre, 2002. Pierre-André Taguieff, longtemps proche de SOS-racisme, affiche ainsi sa distance après la guerre en attaquant « l'antiracisme différencialiste » qui ferait le jeu de l'extrême droite. Taguieff P.-A., Face au racisme, Paris, La Découverte, 1991, notamment tome 2, pp. 13-63.

57.. Wieviorka M. , La France raciste, Paris, Le Seuil, 1992.

58. Bataille P., Le racisme au travail, Paris, La Découverte, 1998.

59.. La technique vient des Etats-Unis et de Grande-Bretagne et consiste à présenter deux candidats aux faciès différents mais de formation, profils et styles similaires. 60.. Une dizaine d'entretiens recueillis dans ce cadre sont consultables sur le site Internet de l'association : www.discriminations-racistes.org/2_victime/recit.htm. 61.. 1997 a été proclamée « année européenne contre le racisme ». En application de l'article 13 du traité d'Amsterdam encourageant le Conseil de l'Union européenne à prendre « les mesures nécessaires en vue de combattre toute discrimination fondée sur le sexe, la race ou l'origine ethnique, la religion ou les convictions, un handicap, l'âge ou l'orientation sexuelle ", deux directives ont été présentées à la Commission le 25 novembre 1999. L'une d'elles invite notamment les Etats à mettre en place un ou plusieurs organismes de promotion de l'égalité de traitement afin notamment de soutenir et d'aider les victimes dans leurs démarches.

62.. Belorgey J.-M., Lutter contre les discriminations, Paris, ministère de l'Emploi et de la solidarité, 1999. Notons que le terme « société d'accueil » employé ici témoigne encore de la tendance du discours politique à enfermer les immigrés et leurs descendants, souvent citoyens français dans la différence. 
63.. Wieviorka M., « Faut-il en finir avec la notion d'intégration? », Les Cahiers de la sécurité intérieure, $\mathrm{n}^{\circ} 45,2001, \mathrm{pp} .9-20$.

64.. Circulaire du Premier ministre du 2 mai 2000.

65.. Adorno S., « Racial Discrimination and Criminal Justice in São Paulo », in Reichmann R. (dir.), Race in contemporary Brazil. From Indifference to inequality, University Park, The Pennsylvania State University Press, 1999, pp. 123-137.

66.. Secretaria de justiça e direitos humanos, Conselho de Desenvolvimento da Communidade Negra, A leie o Direito Do Negro, vol. I, Salvador, novembre 1994.

67.. Comité pour l'élimination des discriminations raciales (CERD), compte-rendu analytique de la 1157ème séance : Bolivia, Brazil, CERD/C/SR.1157, 1996.

68.. Nations Unies, 10 juin 1998, Conseil Economique et Social. Document de travail commun sur l'article 7 de la Convention internationale sur l'élimination de toutes les formes de discrimination raciale. Etabli par M. José Bengoa, M. Ivan Garvalov, M. Mustafa Mehedi et Mme Shanti Sadiq Ali.

69.. Comparative Human Relations Initiative, The Southern Education Foundation, Beyond Racism, Embracing an interdependent future. Brazil, South Africa, The United States, Three nations at the crossroads, Atlanta, The Southern Education Foundation, 2000. 70.. Human Rights in Brazil, The Global Justice Center Annual Report, étude dirigée par Sandra Carvalho, 2002. Cependant, notons que cette mesure est loin d'entraîner des pratiques systématiques au sein des universités. Chaque université est autonome et décide de sa politique propre. Une polémique a éclaté dernièrement à l'université de Brasilia concernant la politique des quotas pour les étudiants de couleur. Parmi les premiers enjeux, celui de la définition de la couleur. Ce sont les phénotypes caractérisant les personnes noires sur lesquels se fondent les universités. Les candidats sélectionnés par le système des quotas doivent attestés avoir des traits qui symbolisent la race (la couleur de la peau, les cheveux, le nez, la bouche). L'université de Brasilia a prévu une phase de vérification des déclarations de couleur faites par les candidats. Les photos sont examinées par une commission spécialement prévue à cet effet. Au vu du métissage complexe de la population brésilienne, cette mesure engendre on s'en doute facilement, d'importantes controverses sur les homologations qui ressortent de l'avis de la commission (Correio Brazilense, 03 janvier 2005). Ces informations sont tirées du site Internet : http://www.observa.ifcs.ufrj.br/sobre/ (Site de l'observatoire des actions de discriminations positives dans l'enseignement universitaire).

71.. Revue Caros Amigos, juin 2002. Traduction : Georges da Costa, octobre 2002

72. Revue Caros Amigos, juillet 2002. Traduction : Georges da Costa, septembre 2002.

73.. « Race in Brazil : Out of Eden », The Economist, 3 juillet 2003.

74.. Poli A., « Le renouvellement de l'action publique contre le racisme dans le cadre du dispositif 114/Codac ", in Cossée C., Lada E., Rigoni I. (dir.), Faire figure d'étranger. Regards croisés sur la production de l'altérité, Paris, Armand Colin, 2004, pp. 211-224. 75.. La loi du 30 décembre 2004 installe cette nouvelle autorité. Concernant les cas de discrimination, l'autorité pourra soit être saisie directement par la victime ou par l'intermédiaire d'un parlementaire ou d'un représentant français au Parlement européen ou encore être saisie conjointement par la victime et une association. Enfin l'autorité pourra également s'auto-saisir, si une victime identifiée ne s'y oppose pas. L'auto-saisine est particulièrement importante en matière de discrimination indirecte, où des pratiques collectives excluant des groupes de personnes peuvent être révélées, sans qu'une intention délictueuse puisse être dégagée et sans qu'on puisse toujours identifier précisément des victimes. 
76.. Adesky J. (d'), Racismes et antiracismes au Brésil, Paris, L'Harmattan, 2001.

77.. Concernant ce mouvement de revendication de l'identité afro-brésilienne, voir notamment le mémoire de maîtrise de sociologie de Fabien Liquori, La réafricanisation de la culture de Bahia par le candomblé, (Directeur : Angelina Peralva), Université Toulouse le Mirail, 2002. Voir également Roth Gordon J., Hip-Hop Brasileiro : Brazilian Youth and Alternative Black consciousness Movements, papier présenté au cours de la rencontre annuelle de la American Anthropology Association, Chicago, 1999

78. Wieviorka M. et al., La tentation antisémite. La haine des Juifs dans la France d'aujourd'hui, Paris, Robert Laffont, 2005.

\section{RÉSUMÉS}

L'idée de démocratie raciale au Brésil et le modèle républicain en France constituent la toile de fond des mouvements de composition et de décomposition de la question du racisme à l'œuvre dans ces deux pays. Ces formules consacrées ne sont pas sans liens avec la manière par laquelle on se représente le racisme et façonnent des impératifs quant à la manière de le combattre. La réflexion croisée sur l'évolution des formes de reconnaissance du racisme au Brésil et en France, que propose cet article, permet notamment de revisiter des conceptions juridiques, politiques, sociales et le vécu personnel qui lui sont attachés à différentes périodes. Des deux côtés de l'Atlantique, les modes de compréhension du racisme ont longtemps fait peser une sorte de déterminisme sur les victimes en les maintenant quelque part à distance du problème.

The Brazilian racial democracy and the French Republican model have determined specific conceptions of racism and different ways to tackle it. Examining racism through these two political contexts leads to raise the question of the link between its legal, political, social and personal définitions. On both sides of the Atlantic, perspectives on racism have made victims passive, keeping them away from the problem. Experiences of racism and racial discriminations have remained in the shadow of history or have melted in the social issue. As time goes by, many great mutations related to the way of thinking differences in Brazil and France have influenced the debate on racism. Public autorities are under increasing pressure to handle the problem and to pay more attention to lived experience of racial discriminations. These evolutions show that the political culture of both countries can no longer contain the fight against racism crossed over global to personal conceptions which challenge the State's action.

\section{INDEX}

Index géographique : Aires géographiques comparées, Brésil, France

Mots-clés : racisme, victimes et victimisation 


\section{AUTEUR}

\section{ALEXANDRA POLI}

Alexandra Poli est docteure en sociologie. Elle est rattachée au CADIS-EHESS-CNRS et a récemment soutenu sa thèse sur la question de l'expérience vécue du racisme et des discriminations raciales en France. 